Original Research Paper

\title{
Multidrug Resistant Uropathogens among Egyptian Pregnant Women
}

\author{
${ }^{1}$ Wafaa Salah Metwally and ${ }^{2}$ Walid Mohamed Elnagar \\ ${ }^{1}$ Department of Medical Microbiology and Immunology, Faculty of Medicine, Zagazig University, Egypt \\ ${ }^{2}$ Department of Obstetrics and Gynecology, Faculty of Medicine, Zagazig University, Egypt
}

\author{
Article history \\ Received: 10-07-2019 \\ Revised: 05-12-2019 \\ Accepted: 19-12-2019 \\ Corresponding Author: \\ Department of Medical \\ Microbiology and \\ Immunology, Faculty of \\ Medicine, Zagazig University, \\ Egypt \\ Tel: 00201006954222 \\ Email: drwafaa.salah@yahoo.com
}

\begin{abstract}
Urinary Tract Infections (UTIs) in pregnancy is a common medical problem associated with significant increase in the risk of fetal morbidity and mortality. The effective appropriate antimicrobial agent is a major concern as many effective antimicrobial groups cannot be used due to their potential toxicity to the fetus as well as the increasing rates of Multidrug-Resistant (MDR) strain isolation. This crosssectional study was conducted on women attending the antenatal care of Zagazig University. This study aimed to estimate prevalence of various uropathogens in UTI among the pregnant women and determine their antimicrobial resistance patterns. Clean catch mid-stream urine samples were obtained, complete microbiological examination and antibiotic susceptibility testing were carried out. More than half of culture positive samples was obtained from asymptomatic pregnant women. Asymptomatic bacteriuria was more common among primigravida, women of low socioeconomic class and with history of previous Cesarean section. Gram negative bacterial isolates were the most prevalent $(71 \%)$. The most frequently isolated pathogens were: $E$. coli $34 \%$, K. pneumoniae $26 \%$ and S. saprophyticus $23 \%$, among the total Gram negative isolates, $95.0 \%$ were MDR. Meropenem was the antibiotic with the highest susceptibility rate $(96 \%)$ on $E$ coli isolates, followed by nitrofurantoin (74\%) and tobramycin (72\%). Recommendations: Urine culture and sensitivity must be the role as MDR pathogens are increasing, rationalization of antibiotic administration is a must.
\end{abstract}

Keywords: MDR, Bacteriuria, Pregnancy

\section{Introduction}

Urinary Tract Infection (UTI) in pregnancy is a major challenge for physicians. The extensive structural and functional changes in the urinary tract during pregnancy increase the risk of UTI. These changes include ureteral dilatation, increased volume of urinary bladder along with decreased its tone, increased urinary stasis and ureterovesical reflux (Duarte et al., 2008). Pregnancy-induced biochemical changes; glucosuria, amino acids and hormone degradation products and $\mathrm{pH}$ changes are also condemned (Jeyabalan and Lain, 2007; Jolley and Wing, 2010).

Urinary tract infection is a clinical condition where inflammatory response of urothelium due to bacterial invasion occurs, which is usually associated with bacteriuria, however, in pregnancy UTIs are classified as symptomatic and asymptomatic bacteriuria (Fasalu Rahiman et al., 2015)

Asymptomatic Bacteriuria (ASB); bacteria present in the urine without evident symptoms, or Symptomatic Bacteriuria (SB); bacteria invade urinary tract tissues inducing an inflammatory response and causing symptoms. Pregnant women with untreated ASB have a significant risk of developing acute pyelonephritis later during pregnancy (Smaill and Vazquez, 2007).

Bacteriuria in pregnancy either asymptomatic or symptomatic is associated with a $50 \%$ increase in the risk of low birth weight and a significant increase in the risk of premature delivery, fetal mortality, chronic renal faluire, pre-eclampsia, hypertension, anemia and postpartum sepsis (Farkash et al., 2012; Dash et al., 2013). The prevalence of UTI in pregnancy is variable worldwide, with a range of 2\%-10 in different parts of 
the world (Mathia et al., 2004; Borchert et al., 2008), however, some countries have higher prevalence (Onoh et al., 2013; Mohamed et al., 2017).

The species of isolated uropathogens are relatively limited, with the eternal leadership of Eschericia coli as a major cause accounting for $75 \%--90 \%$ of uncomplicated UTI isolates. Other players are involved; S. saprophyticus, Klebsiella pneumoniae, Proteus mirabilis and group B Streptococci (Mukherjee et al., 2014; Bose and Revankar, 2018).

With the emergence of drug resistance and particularly the Extended Spectrum Beta Lactamase (ESBL) production by Gram negative bacteria and Methicillin Resistance in Staphylococcus species (MRSA), antibiotic options for treating pregnant woman with bacteruria become limited (Thapa et al., 2015; Bose and Revankar, 2018). Multidrug-Resistant (MDR) strains are defined as acquired non-susceptibility to at least one agent in three or more antimicrobial categories (Magiorakos et al., 2012). In developing countries, where self medication practice and irrational antibiotic use are common, MDR represent a major concern.

This study was conducted to find out the prevalence of various uropathogens in UTI among the pregnant women and their antimicrobial resistance patterns and to determine the prevalence of MDR isolates in these subjects.

\section{Methodology}

\section{Study Design}

A cross-sectional study during the period from January 2018 to January 2019 was conducted on pregnant women attending the antenatal care clinic of Zagazig University Hospital. Participants were excluded if they had antimicrobial drug therapy in the preceding 2 weeks prior to sample collection. Informed written consent was obtained from each patient prior to sample collection. Detailed history highlighting the following items was gathered: Age, complaint, period of gestation, parity and previous caesarian sections. Ethical approval was obtained from Zagazig University IRB committee.

\section{Sample Size}

The required sample size was calculated using single population formula (Charan and Biswas, 2013).

The calculated sample size $(n=304)$ was rounded off to 400 .

\section{Microbiological Specimen Processing}

Participants were instructed to obtain a clean-catch midstream urine samples. Sample were collected in a sterile container and cultured on Cysteine Lactose Electrolyte Deficient agar (CLED) in addition to nutrient agar, using a calibrated drop delivering $0.001 \mathrm{~mL}$ of urine.
Inoculated plates were incubated at $37^{\circ} \mathrm{C}$ degree overnight. The cultured plates were examined and bacterial colonies were counted. Identification of bacterial growth was done depending on cultural morphology and Gram staining. Gram positive cocci were subjected to catalase, coagulase and DNase tests, in cases of Staphylococcal isolates, novobiocine sensitivity was tested and subculture on Mannitol salt agar. Gram negative rods were identified by the following biochemical reaction; oxidase, urease, citrate utilization, indole and fermentative profile on triple sugar agar. Significant bacteriuria was considered when $10^{5}$ colony forming units per $\mathrm{ml}$ urine of one or two isolate (s) was obtained. Wet film examination using high power lens was carried out with reporting of the followings: Pus cells, red blood cells, epithelial cells and crystals.

\section{Antibiotic Susceptibility Testing}

Antimicrobial susceptibility was tested for bacterial isolates by modified Kirby Bauer test Agar. A loop full of bacteria was taken from pure colony and was transferred to a tube containing $5 \mathrm{~mL}$ nutrient broth and mixed gently until it formed a homogenous suspension. The turbidity of the suspension was then adjusted to the optical density of a Mc farland 0.5 tubes measured at $500 \mathrm{~nm}$ absorbance in order to standardize the inoculums size. A sterile cotton swab was then dipped into the suspension and the excess fluid was removed by gentle rotation of the swab against the surface of the tube. Bacterial suspension was evenly distributed over the entire surface of Mueller-Hinton plates using the swab by successive $90^{\circ}$ rotation movement and keeping Petri dish lids in place, the antimicrobial discs were evenly distributed on the inoculated plates. The selection of antibiotic tested is guided by Clinical Laboratory Standard CLSI (2017), US Food and Drug Administration (FDA) categories of medications in pregnancy and the type of isolated bacteria regarding Gram staining. Gram-negative bacterial isolates were tested against the following antimicrobials amoxicillin-clavulanic acid, cefazolin, cefepime, cefotaxime, ceftazidime, ceftraixone, cefuraxime, meropenme, nitrofurantoin, pipracillin tazobactam, tobramycin, trimethoprim-sulphamethoxazole.

Gram positive organisms were tested against the following antimicrobial agents: Azithromycin, erythromycin, clindamycin, cefoxitin, linezolide, trimethoprim-sulphamethoxazole, nitrofurantoin, gentamycin, rifampicin, vancomycin.

Antibiotic discs used were supplied by (Oxoid UK).

The plates were then transferred to incubator at $37^{\circ} \mathrm{C}$ for 18-24 h. Diameter of the zone of inhibition around the disc was measured to the nearest millimeter using a metal caliper and the isolate were classified as sensitive and resistant. (Intermediate agents are considered resistant) (CLSI, 2017). 


\section{ESBL Detection}

Isolates with zone diameters less than $25 \mathrm{~mm}$ for ceftriaxone and less than $22 \mathrm{~mm}$ for ceftazidime were subsequently confirmed for ESBL production via double-disc synergy test (DDST). Amoxy-clavulanic acid $(20 / 10 \mu \mathrm{g})$ disc was placed in the center of ceftazidime $(30 \mu \mathrm{g})$ and cefotaxime $(30 \mu \mathrm{g})$ discs with center to center distance of $25-30 \mathrm{~mm}$. After overnight incubation at $37^{\circ} \mathrm{C}$, confirmation of ESBL producing pathogen was assessed when the zone of inhibition around ceftazidime and cefotaxime was expanded by at least $5 \mathrm{~mm}$ by the presence of clavulanic acid. E. coli ATCC 25922 and Klebsiella pneumoniae ATCC 700603 were used as negative and positive controls for ESBL production, respectively (CLSI, 2017).

\section{MRSA Detection}

Staphylococcal isolates separately tested on additional $\mathrm{MH}$ agar plates against cefoxitin $30 \mathrm{ug}$ and incubated at $33-35^{\circ} \mathrm{C}$; ambient temperature for $24 \mathrm{~h}$ to detect mecA-Mediated Oxacillin Resistance.

\section{Statistical Methods}

Data were analyzed using OpenEpi, Version 3, data expressed in percentage, Chi square test was used to analyze qualitative data. Significant results obtained when $P$ value is $\leq 0.05$.

\section{Results}

Out of the 400 examined urine samples, 182 samples revealed significant bactruria, this amounted to a $45 \%$ positive culture rate. Only $78(43 \%)$ positive samples belonged to participants with UTI symptoms (fever, dysurea, frequency). The remaining 104 (57\%) positive samples were considered as ASB. Socio-demographic and clinical criteria of participants with positive cultures are displayed in table 1.

\section{Bacterial Isolates}

Types and prevalence of isolated organisms are displayed in Table 2. Gram negative pathogens accounted for $71 \%(\mathrm{n}: 130)$ of the isolated organisms (n:182) of positive culture samples. E. coli (62) $34 \%$, K. pneumoniae (48) $26 \%$, S. saprophyticus (40) 23\%, Morganella (9) 5\%, Streptococci (8) 5\%, Pseudomonas (7)3\%, Enterobacter (2) $1 \%$, Enterococci (2)1\%, Proteus (2) $1 \%$, Methicillin Resistant S. aureus (MRSA) (2) $1 \%$.

\section{Antibiotic Resistance Pattern}

Regarding Gram negative isolates, meropenem was the antibiotics with maximum sensitivity (Fig. S1), about $96 \%$ of the isolated $E$ coli was meropenem sensitive, susceptibility rate of $E$ coli isolates toward other agents was as follow: Nitrofurantoin (74\%), tobramycin (72\%), Azteronam (68\%), cefepime (63\%), ceftriaxone (55\%). $K$. pneumoniae species showed high resistance rates (80$100 \%$ ) to most tested agents, azteronam showed the highest susceptibility rate in such isolates. Among the total Gram negative isolates, 95\% (124/130) were MDR. There were no isolates sensitive to all antibiotics tested (Table S1 and Fig. S2).

Prevalence of ESBL producing gram negative isolates demonstrated in Table 3.

Twenty nine (47\%) isolates of Escherichia coli and $21(44 \%)$ isolates of Klebsiella pneumoniae were ESBL producers (Figure 1) The total ESBL producing isolates among gram negative pathogens was 50 (38\%).

In isolates of some Gram negative bacteria (Morganella, Pseudomonas, Proteus, Enterobacter), $100 \%$ resistance rates were detected toward some agents; amoxicillin-clavulanic acid, cefazolin, cefepime, ceftazidime (Fig. 2S).

Table 1: Socio-demographic, obstetric and clinical variables of pregnant women with culture positive results

\begin{tabular}{|c|c|c|c|c|c|c|}
\hline & SB No: 78 & & ASB No: 104 & & $\mathrm{X}^{2}$ & $\mathrm{P}$ \\
\hline Parity & & & & & 9.7 & $0.007 *$ \\
\hline Primigravida & 20 & $26 \%$ & 56 & $54 \%$ & & \\
\hline Primipara & 31 & $40 \%$ & 37 & $36 \%$ & & \\
\hline Multipara & 27 & $34 \%$ & 11 & $10 \%$ & & \\
\hline Age & & & & & 3.6 & 0.16 \\
\hline $19-27$ & 23 & $29 \%$ & 21 & $20 \%$ & & \\
\hline 28- 35 & 37 & $48 \%$ & 47 & $45 \%$ & & \\
\hline $36-43$ & 18 & $23 \%$ & 36 & $35 \%$ & & \\
\hline Previous CS & & & & & 4.6 & $0.03 *$ \\
\hline Yes & 46 & $59 \%$ & 77 & $74 \%$ & & \\
\hline No & 32 & $41 \%$ & 27 & $26 \%$ & & \\
\hline Socioeconomic state & & & & & 2.4 & 0.2 \\
\hline Low & 45 & $58 \%$ & 71 & $68 \%$ & & \\
\hline Moderate & 21 & $27 \%$ & 19 & $18 \%$ & & \\
\hline High & 12 & $15 \%$ & 14 & $14 \%$ & & \\
\hline
\end{tabular}


Wafaa Salah Metwally and Walid Mohamed Elnagar / American Journal of Infectious Diseases 2019, 15 (4): 115.122 DOI: 10.3844/ajidsp.2019.115.122

Table 2: Types and prevalence of bacterial isolates

\begin{tabular}{lccr}
\hline Organism & SB (78) & ASB (104) & Total (182) \\
\hline E. coli & 23 & 39 & 62 \\
K. pneumonia & 17 & 31 & 48 \\
S. saprophyticus & 13 & 27 & 40 \\
Morganella & 7 & 2 & 9 \\
Streptococci & 7 & 1 & 8 \\
Pseudomonas & 5 & 2 & 7 \\
Enterobacter & 1 & 1 & 2 \\
Proteus & 1 & 1 & 2 \\
Enterococci & 2 & 0 & 2 \\
MRSA & 1 & 1 & 2 \\
Total & 78 & 104 & 182 \\
\hline
\end{tabular}

Table 3: Prevalence of ESBL producing gram negative isolates

\begin{tabular}{lcc}
\hline Organism & Number $(\%)$ & ESBL producer $(\%)$ \\
\hline E. coli & 62 & $29(47 \%)$ \\
K. pneumonia & 48 & $21(44 \%)$ \\
Morganella & 9 & 0 \\
Pseudomonas & 6 & 0 \\
Enterobacter & 2 & 0 \\
Proteus & 2 & 0 \\
Total & 130 & $50(38 \%)$ \\
\hline
\end{tabular}

Table 4: Antibiogram of the most prevalent Gram positive isolates

\begin{tabular}{|c|c|c|c|c|}
\hline \multirow[b]{2}{*}{ Agent } & \multicolumn{2}{|l|}{ CON n: 42} & \multicolumn{2}{|c|}{ Streptococci n: 8} \\
\hline & $\mathrm{R}$ & $\mathrm{S}$ & $\mathrm{R}$ & $\mathrm{S}$ \\
\hline $\mathrm{AZM}$ & $32(76 \%)$ & $10(34 \%$ & $4(50 \%)$ & $4(50 \%)$ \\
\hline $\mathrm{E}$ & $38(90 \%)$ & $4(10 \%)$ & $6(75 \%)$ & $2(25 \%)$ \\
\hline Clind & $26(62 \%)$ & $16(38 \%)$ & $8(100 \%)$ & $0(0 \%)$ \\
\hline Ceph & $36(86 \%)$ & $6(14 \%)$ & $6(75)$ & $2(25 \%)$ \\
\hline FEP & $36(86 \%)$ & $6(14 \%)$ & $5(62 \%)$ & $3(38 \%)$ \\
\hline FOX & $24(57 \%)$ & $18(43 \%)$ & $3(38 \%)$ & $5(62 \%)$ \\
\hline $\mathrm{LiZ}$ & $2(5 \%)$ & $40(95 \%)$ & $0(0 \%)$ & $8(100 \%)$ \\
\hline SXT & $34(81 \%)$ & $8(19 \%)$ & $7(88 \%)$ & $1(12 \%)$ \\
\hline $\mathrm{F}$ & $10(23 \%)$ & $32(77 \%)$ & $7(88 \%)$ & $1(12 \%)$ \\
\hline Mem & $4(10 \%)$ & $38(90 \%)$ & $1(12 \%)$ & $7(88 \%)$ \\
\hline Gen & $26(62 \%)$ & $16(38 \%)$ & $5(63 \%)$ & $3(37 \%)$ \\
\hline Rif & $14(33 \%)$ & $28(67 \%)$ & $7(88 \%)$ & $1(12 \%)$ \\
\hline
\end{tabular}

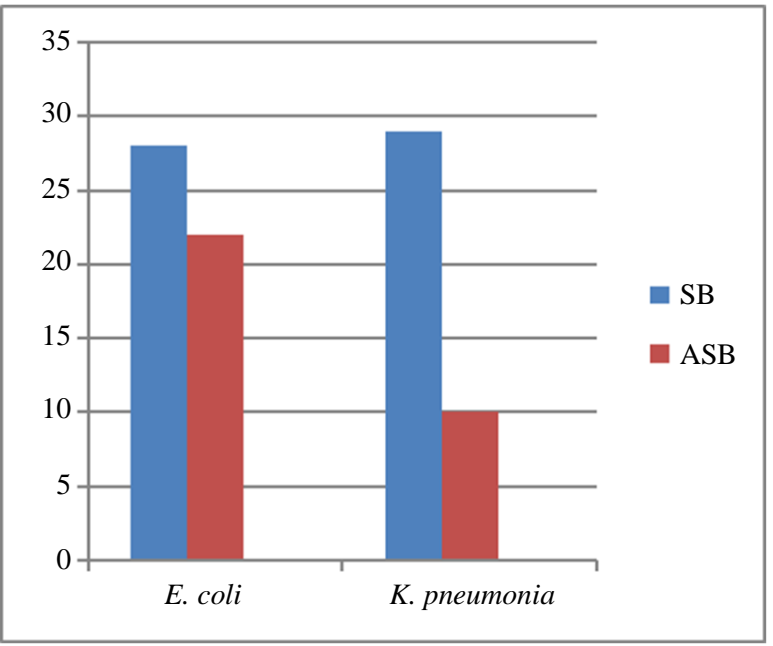

Fig. 1: Distribution of ESBL and non ESBL isolates among SB and ASB, chi square: 0.64, P: 0.2 
Linezolid showed the maximum sensitivity in Coagulase negative staphylococci $(\mathrm{CON})$ isolates, while erythromycin showed the highest resistance rates, however, streptococcal isolates were noticed to have more resistance to clindamycine than erythromycin. High levels of single and multiple antimicrobial resistances against commonly prescribed drugs were observed (Table 4).

\section{Discussion}

The effective appropriate antimicrobial agent is a problematic issue in pregnancy as many antimicrobial groups cannot be used; quinolones, tetracylines due to their potential toxicity to the fetus (MatuszkiewiczRowińska et al., 2015). With the emergence of drug resistance and particularly the ESBL production by Gram negative bacteria and methicillin resistance in Staphylococcus species, antibiotic options for treating pregnant woman with bacteruria are limited (Thapa et al., 2015; Bose and Revankar, 2018).

In the current study, a $45 \%$ positive culture rate was reported, this is a higher rate than those obtained from previous studies carried out by different researchers in the same governorate (Dimetry et al., 2007), the prevalence of laboratory confirmed UTI in pregnant was estimated to be $30.29 \%$ in neighboring regions (Suez governorate) in Egypt (Mohamed et al., 2017). Similar findings were obtained in Yemen and Bangaldish (Almushait et al., 2013), however Indian (Manjula et al., 2013) and Nigerian (Onoh et al., 2013) studies reported slightly raised rates (49.4\% and $46.5 \%$ respectively). This variation may be explained by the differences in characteristics of study populations, environmental conditions and methodology.

In the this study, more than half of culture positive samples was obtained from asymptomatic pregnant women. Asymptomatic bacteriuria in pregnancy was more common among primigravida (54\%) compared to multigravida, however, Dash et al. (2013), reported that majority $(54.4 \%)$ of the subjects they tested were multipara. This discrepancy could be explained by absence of routine urine culture in Egypt, so primigravida especially in rural communities are more prone to ASB. These findings highlight the importance of urine culture in the antenatal care practice to pick up the affected pregnants and giving the appropriate therapy.

Previous Caesarian section was associated with higher risk of ASB (74\%), The past decade had witnessed a great increase in the prevalence of CS in Egypt (52\%), which suggests that cesarean delivery might be overused or used for inappropriate indications (The Population Council, 2018).

ASB was more prevalent in women of low socioeconomic class $(68 \%)$ although not statically significant. These findings are similar to those obtained by (Kerure et al., 2012; Bose and Revankar, 2018).

Gram-negative bacteria were more prevalent with a noticed predominance of $E$. coli and $K$. pneumonia. Similar findings have been reported in Ethiopia (Derese et al., 2016). This is a cardinal finding that had been previously noted by many other studies in different countries (Moyo et al., 2010; Dash et al., 2013; Onoh et al., 2013). As E. coli have a normal presence in the bowel, infection could occur by contamination of the urethra by fecal matter due to poor and difficult hygiene, the anatomical proximity to the genito-urinary area in womans and the urinary stasis induced by pregnancy (Farkash et al., 2012; Huttner et al., 2015). About 38\% of Gram negative isolates was ESBL producers, this ratio is higher than those obtained by other authors Iqbal et al. (2014; Thapa et al., 2015). This higher rate could be explained by the irrational antibiotic use and selfmedication practice especially with broad spectrum antibiotics that could be easily obtained in many regions in Egypt without medical prescriptions.

Most Gram negative isolates were resistant to more than three antibiotic classes. The antibiotic susceptibility patterns of Gram Negative bacteria showed that meropenem, azreonam and nitrofurantoin were effective in more than half of these isolates. These findings are in agreement with an Indian study (Bose and Revankar, 2018) stated that meropenem was the most sensitive antibiotic (84\%), followed by cefaperazone $(73.1 \%)$ and nitrofurantoin $(69.2 \%)$. The antibiotic with the overall highest sensitivity pattern in this study was meropenem, however, nitrofurantoin showed an appreciated sensitivity rates in Proteus, morganella, E coli and CoNs, these findings are quite similar to those obtained by (Onoh et al., 2013), so nitrofurantoin could be a good option in the management of UTI in pregnancy, especially when the isolated organism has good sensitivity to nitrofurantoin. Results from systematic review and meta-analysis of 27 controlled clinical trials revealed that nitrofurantoin appears to have good clinical and microbiological efficacy for UTI caused by common uropathogens, with clinical cure rates varying between $79 \%$ and $92 \%$ (Huttner et al., 2015).

Ceftazidime, cephazolin and amoxicillinclavulanic acid had the higher resistance rates. Similar findings was reported by Bose and Revankar (2018). The resistance rates of amoxicillin-clavulanic acid in Gram negative isolates were found to be $65-100 \%$, with overall rate of $82 \%$, this could be explained by the irrational use of the drug due to its easy rout of administration and relatively low price.

Among the total Gram negative isolates, 95.0\% (124/130) were MDR, this figure is higher than that obtained by Thapa et al. (2015) in Nepal (72\%), however other studies revealed higher isolation rate of MDR (Alemu et al., 2012; Gessese et al., 2017). The high prevalence of MDR reported in our study might be due 
to the easily obtained, non-prescribed drugs. Although bacterial resistance is a natural phenomenon, its current high and dangerous rate usually is related to the misuse of antimicrobial drugs, failure to adhere to standard treatment guideline and inadequate or absence of stringent antimicrobial drug resistance surveillance program.

\section{Conclusion}

Multidrug resistant bacteria is a major health problem especially among pregnant woman. Urine culture and sensitivity must be included in the antenatal care practice to detect ASB and administer accurate treatment.

\section{Acknowledgement}

We thank our nurse staff that conferred great support in sampling and patient communication.

\section{Funding Information}

This research did not receive any specific grant from funding agencies in the public, commercial, or not-forprofit sectors.

\section{Author's Contributions}

Wafaa Salah Metwally: Study idea and design, microbiological processing of the specimen, analysis of laboratory data, writing and editing the manuscript, corresponding author.

Walid Mohamed Elnagar: Patient selection and history taking, analysis of clinical data, writing and editing the manuscript.

\section{References}

Abdel-Tawab, A., D. Oraby, N. Hassanein and S. ElNakib, 2018. Cesarean section deliveries in Egypt: Trends, practices, perceptions and cost. Cairo: Population Council.

Alemu, A., F. Moges, Y. Shiferaw, K. Tafesse and A. Kassu et al., 2012. Bacterial profile and drug susceptibility pattern of urinary tract infection in pregnant women at University of Gondar Teaching Hospital, Northwest Ethiopia. BMC Res. Notes, 5: 197-197. DOI: 10.1186/1756-0500-5-197

Almushait, M.A., H.A. Mohammed, D.A. Al-Harthy and A.M. Abdullah, 2013. Prevalence and predisposing factors of urinary tract infections among pregnant women in Abha general hospital. Int. J. Basic Sci. Applied Res., 11: 18-29.

Borchert, D., L. Sheridan, A. Papatsoris, Z. Faruquz and J.M. Barua et al., 2008. Prevention and treatment of urinary tract infection with probiotics: Review and research perspective. Indian J. Urol., 24: 139-144. DOI: $10.4103 / 0970-1591.40604$
Bose, A.M. and V.M. Revankar, 2018. Proportion of pregnancy associated asymptomatic bacteriuria in a tertiary care hospital. Int. J. Reproduct. Contracept. Obstetr. Gynecol., 7: 952-956.

DOI: $10.18203 / 2320-1770$. ijrcog20180872

Charan, J. and T. Biswas, 2013. How to calculate sample size for different study designs in medical research? Indian J. Psychol. Med., 35: 121-126. DOI: $10.4103 / 0253-7176.116232$

CLSI, 2017. Clinical laboratory standard. M100.

Dash, M., S. Sahu, I. Mohanty, M.V. Narasimham and J. Turuk et al., 2013. Prevalence, risk factors and antimicrobial resistance of asymptomatic bacteriuria among antenatal women. Basic Clin. Reproductive Sci., 2: 92-96.

Derese, B., H. Kedir, Z. Teklemariam, F. Weldegebreal and S. Balakrishnan, 2016. Bacterial profile of UTI and antimicrobial susceptibility pattern among pregnant women attending at antenatal Clinic in Dil Chora Referral Hospital, Dire Dawa, eastern Ethiopia. Therapeutic Clin. Risk Manage., 12: 251-260.

Dimetry, R.S., H.M. El-Tokhy, N.M. Abdo, M.A. Ebrahim and M. Eissa, 2007. Urinary tract infection and adverse outcome of pregnancy. J. Egypt Public Health Assoc., 82: 203-218. PMID: 18410708

Duarte, G., A.C. Marcolin, S.M. Quintana and R.C. Cavalli, 2008. Urinary tract infection in pregnancy. Rev. Brazilian Gynecol. Obstetr., 30: 93-100. PMID: 19142482

Farkash, E., A.Y. Wientraub, R. Sergienko, A. Wiznitzer and A. Zalotnik et al., 2012. Acute antepartum pyelonephritis in pregnancy: A critical analysis of risk factors and outcomes. Eur. J. Obstetrica Gynecol. Reproductive Biol., 162: 24-7. PMID: 22381037

Fasalu Rahiman, O.M., T. Balasubramanian, M. Shejina and M. Musambil, 2015. A review on urinary tract infection in pregnancy. Int. J. Pharma Res. Rev.

Gessese, Y.A., D.L. Damessa, M.M. Amare, Y.H. Bahta and A.D. Shifera et al., 2017. Urinary pathogenic bacterial profile, antibiogram of isolates and associated risk factors among pregnant women in Ambo town, Central Ethiopia: A cross-sectional study. Antimicrobial Resistance Infect. Control, 6: 132-132. DOI: $10.1186 / \mathrm{s} 13756-017-0289-6$

Huttner, A., M. Verhaegh, S. Harbarth, A. Muller and U. Theuretzbacher et al., 2015. Nitrofurantoin revisited: A systematic review and meta-analysis of controlled trials. J. Antimicrobial Chemotherapy, 70: 5-5. DOI: 10.1093/jac/dkv147 
Iqbal, R., A. Majid, I.A. Alvi, A. Hayat and F. Andaleeb et al., 2014. Multiple drug resistance and ESBL production in bacterial urine culture isolates. Am. J. BioScience, 2: 5-12.

DOI: $10.11648 /$ j.ajbio.20140201.12

Jeyabalan, A. and K.Y. Lain, 2007. Anatomic and functional changes of the upper urinary tract during pregnancy. Urolol. Clin. North Am., 34: 1-6.

DOI: 10.1016/j.ucl.2006.10.008

Jolley, J.A. and D.A. Wing, 2010. Pyelonephritis in pregnancy: An update on treatment options for optimal outcomes. Drugs, 70: 1643-55.

DOI: $10.2165 / 11538050-000000000-00000$

Kerure, S.B., R. Surpur, S.S. Sagarad, and S. Hegadi, 2013. Asymptomatic bacteriuria among pregnant women. Int. J. Reproduct. Contracept. Obstetr. Gynecol., 2: 213-216

DOI: $10.5455 / 2320-1770 . i j r \operatorname{cog} 20130621$

Magiorakos, A.P., A. Srinivasan, R.B. Carey, Y. Carmeli and M.E. Falagas et al., 2012. Multidrug-resistant, extensively drug-resistant and pandrug-resistant bacteria: An international expert proposal for interim standard definitions for acquired resistance. Clin. Microbiol. Infect., 18: 268-281.

DOI: 10.1111/j.1469-0691.2011.03570.x

Manjula, N.G., C.M. Girish, A.P. Shripad, M.G. Subashchandra and T.S. Channappa, 2013. Incidence of urinary tract infections and its aetiological agents among pregnant women in Karnataka region. Adv. Microbiol., 3: 473-478. DOI: 10.4236/aim.2013.36063

Mathia, E., R.J. Thomas, S. Chandy, M. Mathai and S. Bergstrom, 2004. Antimicrobials for the treatment of urinary tract infection in pregnancy: Practice in southern India. Pharmacoepidermiol. Drug Safety, 13: 645-652. DOI: $10.1002 /$ pds.950
Matuszkiewicz-Rowińska, J., J. Małyszko and M. Wieliczko, 2015. Urinary tract infections in pregnancy: Old and new unresolved diagnostic and therapeutic problems. Archive Med. Sci., 11: 67-77. DOI: 10.5114/aoms.2013.39202

Mohamed, N.R., H.H. Omar and I.M. Abd-Allah, 2017. Prevalence and risk factors of urinary tract infection among pregnant women in Ismailia City, Egypt. J. Nurs. Health Sci., 6: 62-72.

DOI: 10.9790/1959-0603076272

Moyo, S.J., S. Aboud, M. Kasubi and S.Y. Maselle, 2010. Bacterial isolates and drug susceptibility patterns of urinary tract infection among pregnant women at Muhimbili National Hospital in Tanzania. Tanzan J. Health Res., 12: 236-240. PMID: 24409630

Mukherjee, K., S. Golia, C.L. Vasudha, Babita and D. Bhattacharjee et al., 2014. A study on asymptomatic bacteriuria in pregnancy: Prevalence, etiology and comparison of screening methods. Int. J. Res. Med. Sci., 2: 1085-1091.

Onoh, R.C., O.U.J. Umeora, V.E. Egwuatu, P.O. Ezeonu and T.J.P. Onoh, 2013. Antibiotic sensitivity pattern of uropathogens from pregnant women with urinary tract infection in Abakaliki, Nigeria. Infect. Drug Resistance, 6: 225-233. DOI: $10.2147 /$ IDR.S46002

Smaill, F. and J.C. Vazquez, 2007. Antibiotics for asymptomatic bacteriuria in pregnancy. Cochrane Database Systematic Rev., 18: CD000490-CD000490. DOI: 10.1002/14651858.CD000490.pub3

Thapa, R., P. Lamichhane, M.G. Banjara and G.P. Achary, 2015. Prevalence of extended spectrum beta lactamase producing uropathogens in pregnant women. Asian J. Pharmaceutical Clin. Res., 8: 207-210.

\section{Supplement Material}

Table S1: Antibiogram of Gram negative isolates

\begin{tabular}{|c|c|c|c|c|c|c|c|c|c|c|c|c|c|}
\hline \multirow[b]{2}{*}{ Antibiotic } & \multicolumn{2}{|c|}{ E. coli $\mathrm{n}: 62$} & \multicolumn{2}{|c|}{ K. pneumoniae n: 48} & \multicolumn{2}{|c|}{ Morganella n: 9} & \multicolumn{2}{|c|}{ Pseudomonas n: 7} & \multicolumn{2}{|c|}{ Proteus n:2 } & \multicolumn{2}{|c|}{ Enterobacter $\mathrm{n}: 2$} & \multirow{2}{*}{$\begin{array}{l}\text { Total n: } 130 \\
\text { Resistant isolates } \\
\text { to each antibiotic }\end{array}$} \\
\hline & $\mathrm{R}$ & $\mathrm{S}$ & $\mathrm{R}$ & $\mathrm{S}$ & $\mathrm{R}$ & $\mathrm{S}$ & $\mathrm{R}$ & $\mathrm{S}$ & $\mathrm{R}$ & $\mathrm{S}$ & $\mathrm{R}$ & $\mathrm{S}$ & \\
\hline AMC & $40(65 \%)$ & $22(35 \%)$ & $46(96 \%)$ & $2(4 \%)$ & $9(100 \%)$ & $0(0 \%)$ & $7(100 \%)$ & $0(0 \%)$ & $2(100 \%)$ & $0(\%)$ & $2(100 \%)$ & $0(0 \%)$ & $106(81 \%)$ \\
\hline $\mathrm{CZ}$ & $54(87 \%)$ & $8(13 \%)$ & $48(100 \%)$ & $0(0 \%)$ & $9(100 \%)$ & $0(0 \%)$ & $7(100 \%)$ & $0(0 \%)$ & $2(100 \%)$ & $0(0 \%)$ & $2(100 \%)$ & $0(0 \%)$ & $122(94 \%)$ \\
\hline FEP & $23(37 \%)$ & $39(63 \%)$ & $43(90 \%)$ & $5(10 \%)$ & $9(100 \%)$ & $0(0 \%)$ & $7(100 \%)$ & $0(0 \%)$ & $2(100 \%)$ & $0(0 \%)$ & $2(100 \%)$ & $0(0 \%)$ & $86(66 \%)$ \\
\hline CAZ & $56(90)$ & $6(10 \%)$ & $48(100 \%)$ & $0(0 \%)$ & $9(100 \%)$ & $0(0 \%)$ & $7(100 \%)$ & $0(0 \%)$ & $2(100 \%)$ & $0(0 \%)$ & $2(100 \%)$ & $0(0 \%)$ & $124(95 \%)$ \\
\hline CRO & $28(45 \%)$ & $34(55 \%)$ & $39(81)$ & $9(19 \%)$ & $6(66 \%)$ & $3(34 \%)$ & $7(100 \%)$ & $0(0 \%)$ & $1(50 \%)$ & $0(0 \%)$ & $2(100 \%)$ & $0(0 \%)$ & $82(63 \%)$ \\
\hline CTX & $39(63 \%)$ & $23(37 \%)$ & $37(77 \%)$ & $11(23 \%)$ & $8(88 \%)$ & $1(12 \%)$ & $7(100 \%)$ & $0(0 \%)$ & $2(100 \%)$ & $0(0 \%)$ & $2(100 \%)$ & $0(0 \%)$ & $94(72 \%)$ \\
\hline MEM & $4(6 \%)$ & $58(94 \%)$ & $40(83 \%)$ & $8(17 \%)$ & $0(0 \%)$ & $100(100 \%)$ & $7(100 \%)$ & $0(0 \%)$ & $0(0 \%)$ & $2(100 \%)$ & $1(50 \%)$ & $1(50 \%)$ & $51(39 \%)$ \\
\hline $\mathrm{AZT}$ & $20(32 \%)$ & $40(68 \%)$ & $30(63)$ & $18(37 \%)$ & $2(22 \%)$ & $7(78 \%)$ & $5(71 \%)$ & $2(29 \%)$ & $0(0 \%)$ & $2(100 \%)$ & $2(100 \%)$ & $0(0 \%)$ & $58(45 \%)$ \\
\hline F & $16(26 \%)$ & $46(74 \%)$ & $35(73 \%)$ & $13(27 \%)$ & $6(66 \%)$ & $3(34 \%)$ & $4(57 \%)$ & $3(43 \%)$ & $0(0 \%)$ & $2(100 \%)$ & $2(100 \%)$ & $0(0 \%)$ & $63(48 \%)$ \\
\hline TAZ & $30(49 \%)$ & $32(51 \%)$ & $43(90 \%)$ & $5(10 \%)$ & $8(88 \%)$ & $1(12 \%)$ & $4(57 \%)$ & $3(43 \%)$ & $1(50 \%)$ & $1(50 \%)$ & $2(100 \%)$ & $0(0 \%)$ & $88(68 \%)$ \\
\hline TOB & $17(28 \%)$ & $45(72 \%)$ & $42(88 \%)$ & $6(12 \%)$ & $6(66 \%)$ & $3(34 \%)$ & $5(83 \%)$ & $2(17 \%)$ & $0(0 \%)$ & $2(100 \%)$ & $0(0 \%)$ & $2(100 \%)$ & $70(54 \%)$ \\
\hline SXT & $45(72 \%)$ & $17(28 \%)$ & $44(92 \%)$ & $4(8 \%)$ & $6(66 \%)$ & $3(34 \%)$ & $7(100 \%)$ & $0(0 \%)$ & $0(0 \%)$ & $2(100 \%)$ & $2(100 \%)$ & $0(0 \%)$ & $103(79 \%)$ \\
\hline
\end{tabular}




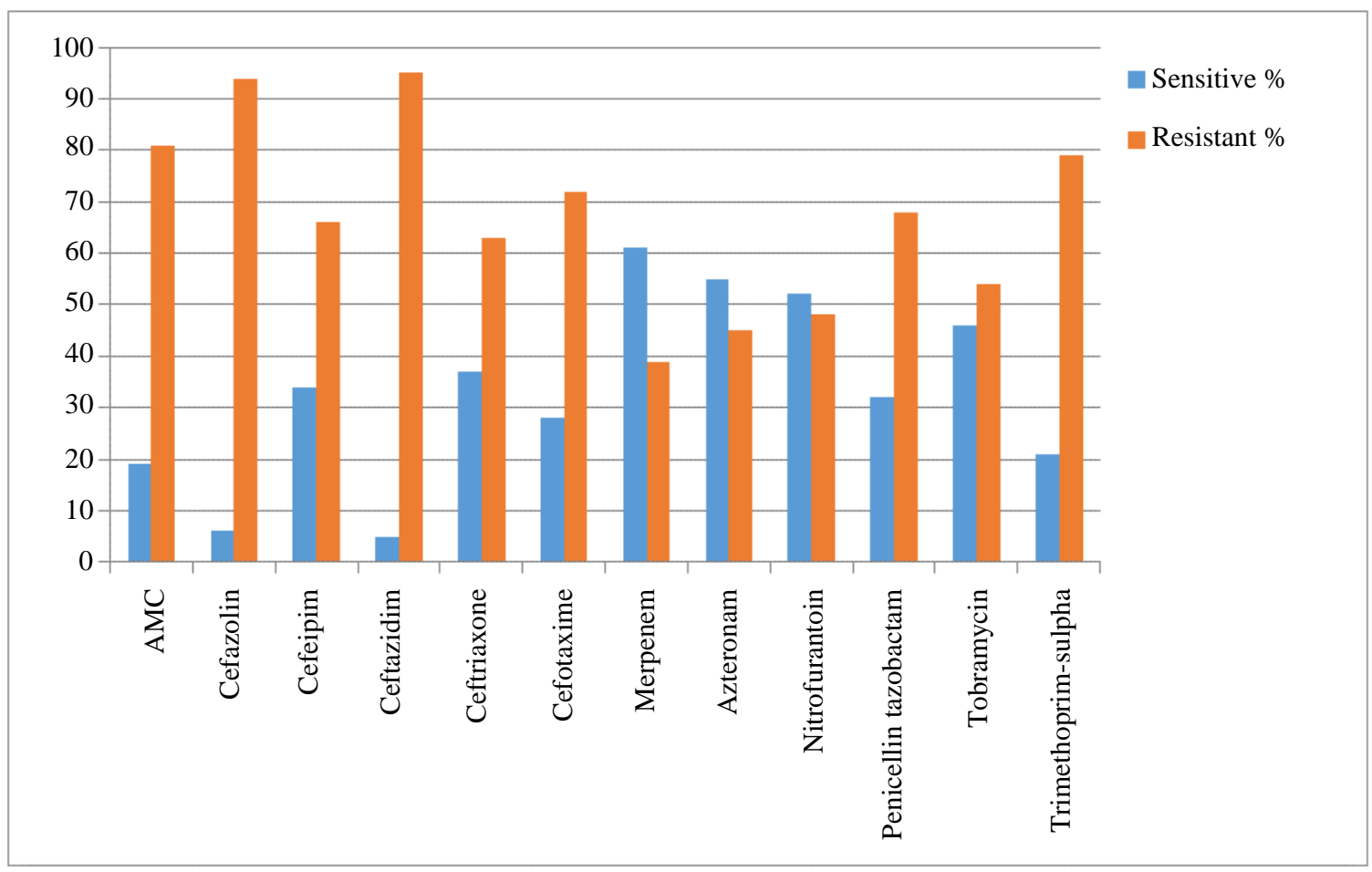

Fig. S1: Percentage of sensitive and resistant isolates toward antibiotics

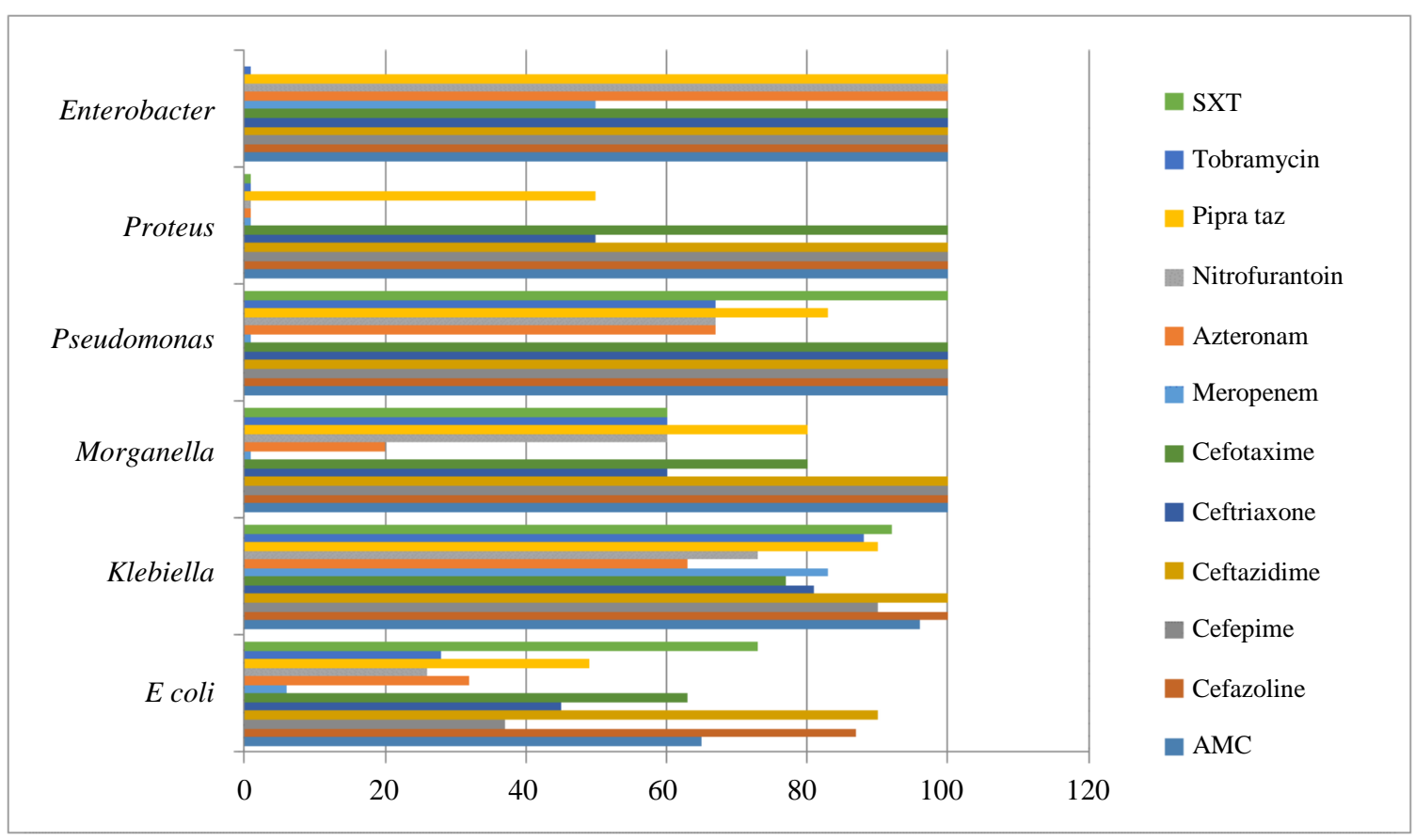

Fig. S2: The resistance rate of individual Gram negative bacteria to the tested antimicrobials 